\title{
STUDIES ON VARIATION OF TRYPTOPHAN METABOLITES LEVEL IN RABBIT PLASMA WITH PREPARATION PROCEDURES BY HIGH PERFORMANCE LIQUID CHROMATOGRAPHY
}

\author{
Ikue MORITA, Tsutomu MASUJIMA, Hisanobu YOSHIDA ${ }^{\circledR}$, and Hideo IMAI \\ Institute of Pharmaceutical Sciences, Hiroshima University School of \\ Medicine, 1-2-3, Kasumi, Minami-ku, Hiroshima-shi, Hiroshima 734, \\ Japan
}

\begin{abstract}
Reversed phase high performance liquid chromatography with direct injection of plasma samples was applied to evaluate the variation of plasma tryptophan ( $T r p)$ metabolites level with sample preparation procedures. Fresh heparinized rabbit plasma showed the peaks corresponding to serotonin (5-HT), 5-hydroxy indole-3-acetic acid (5HIAA), indole-3-acetic acid (IAA), indole-3-lactic acid (ILA), indole3-propionic acid (IPA), kynurenine (Kyn), kynurenic acid (KA) in addition to tryptophan (Trp). By standing plasma at $37^{\circ} \mathrm{C}, 5-\mathrm{HT}$ trended to decrease about $5 \% / h$, but other metabolites levels were constant for at least $24 \mathrm{~h}$. By standing platelet rich plasma (PRP) for $6 \mathrm{~h}, 5-\mathrm{HT}$ and 5-HIAA increased. In addition, 5-hydroxy tryptophol (5-OH TrPOL), which was not found as a common metabolite in fresh plasma, was detected. By standing whole blood for $6 \mathrm{~h}$, almost all metabolites except IAA and IPA increased by several times. Serum levels of 5-HIAA, 5-OH TrPOL and especially 5-HT were higher than that of plasma. Consequently, the plasma samples should be prepared just after collecting blood for the analysis of Trp-metabolites level.
\end{abstract}

Tryptophan (Trp) is metabolized by two major pathways, either through kynurenine or through a series of indoles, as shown in Fig. 1. Many of these metabolites are biologically active, and the importance of Trp and its metabolites in both normal and pathological states has been reviewed ${ }^{1}{ }^{2}$ ). However, there are two major difficulties for the analysis of minute Trp-metabolites in biological samples. First, there is no suitable method concurrently useful for both metabolites through kynurenine and a series of indoles pathways. Secondly, Trp-metabolites level might be varied during a sample preparation step; e.g., 5-HT could be released from platelets or enzymatically converted.

We have reported that high performance liquid chromatography (HPLC) with direct plasma injection has some advantages over a conventional HPLC ${ }^{3) 4}$. For example, increasing of sample volume and accurate total amount analysis (free + bound to plasma proteins) are possible. 
Recently, we have modified our previous method 4 for the enrichment analysis of both metabolites through kynurenine and a series of indoles pathways in plasma ${ }^{5)}$. Using this method, we studied the variation of Trp-metabolites in rabbit heparinized plasma with sample preparation procedures.

\section{MATERIALS AND METHOD \\ Chemicals}

Trp-metabolites were

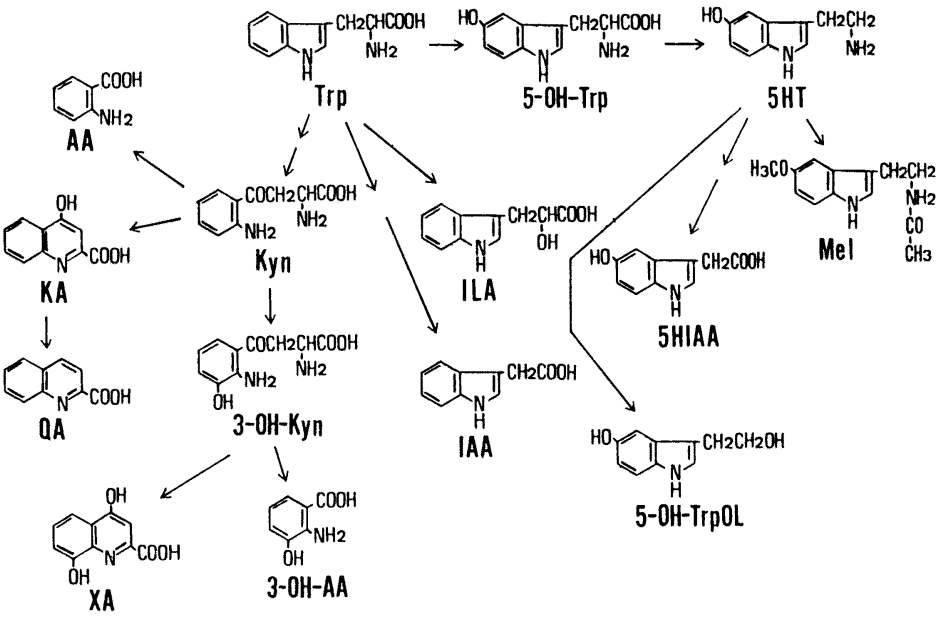

Fig. l Some tryptophan metabolites

obtained from Sigma Chemical

(St. Louis, U.S.A.). All other chemicals including components of elution solvents for HPLC were of guaranteed grade from Yoneyama Pharmaceutical (Osaka, Japan), and were used as received.

\section{Sample preparation}

Blood was drawn into a plastic syringe with or without heparin as an anticoagulant. The blood without anti-coagulant was allowed to clot at room temperature for $20 \mathrm{~min}$, then centrifuged at $1500 \times \mathrm{g}$ for $10 \mathrm{~min}$ at room temperature. The supernatant was taken out and used as serum. Blood with heparin $(0.1 \mathrm{mg} / \mathrm{ml}) \mathrm{was}$ centrifuged at $150 \times \mathrm{g}$ for $15 \mathrm{~min}$ at room temperature to sediment blood cel ls except platelets. The upper phase suspension was taken out and used as platelet rich plasma (PRP). Blood with heparin was centrifuged at $1500 \times \mathrm{g}$ for $10 \mathrm{~min}$ at room temperature, and the supernatant was taken out and used as plasma.

\section{Incubation}

Plasma, PRP or whole blood was put into plastic tubes, and incubated (stood) for 2-24 h at $37^{\circ} \mathrm{C}$. After the incubation, PRP and whole blood were centrifuged at 1500 $\times \mathrm{g}$ for $10 \mathrm{~min}$ at room temperature to obtain plasma. One hundred $\mu l$ of plasma sample was directly injected to HPLC to analyze the Trp-metabolites level.

\section{HPLC apparatus}

Two columns were used; one was a $40 \mathrm{~mm} \times 4 \mathrm{~mm}$ I.D. pre-column packed with protein-coated ODS ${ }^{3 / 6)}$ for enrichment of Trp-metabolites in plasma and also for deproteinization (plasma proteins were eluted out with initial aqueous solution). The other column was a $150 \mathrm{~mm} \times 4.6 \mathrm{~mm}$ I.D. column packed with native oDS (Chemcosorb ODS-H, Chemco, Osaka, Japan) for the separation of Trp-metabolites ${ }^{4}$.

An HPLC apparatus with stepwise elution was assembled in our laboratory. The apparatus, as shown in Fig. 2, consisted of two pumps (Pumps A and B). Flow direction was controlled by a model 7125 sample injection valve (Rheodyne,C.A., 
U.S.A.), a model 7010 flow direction switching valve (Rheodyne), a model $8 \mathrm{~V}$ solvent selector (kyowa seimitsu, Tokyo, Japan) and a sequence programmer SCY-PO lOmron, Tokyo, Japan).

Metabolites in indole pathways were detected by native fluorescence (Ex. 287 $\mathrm{nm}, \mathrm{Em} .340 \mathrm{~nm}$ ) with a model RF-530 fluorometer (Shimadzu, Kyoto, Japan). The indole compounds were also detected by an electrochemical

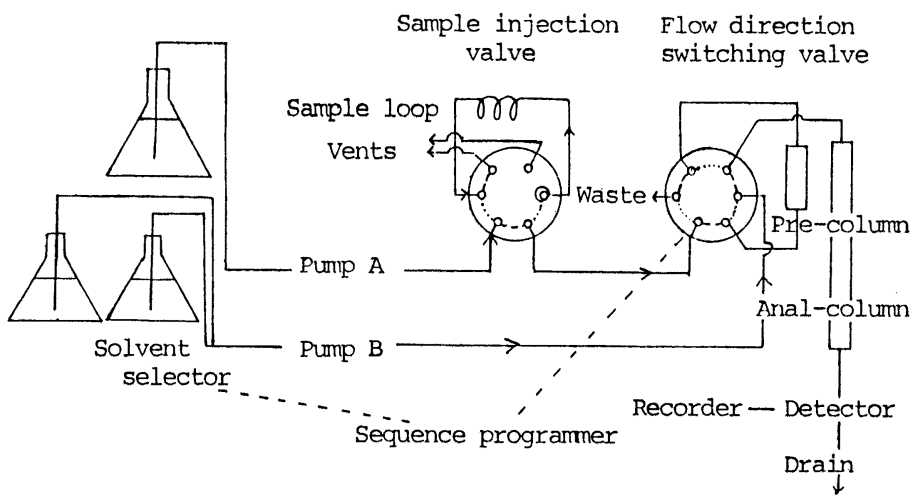

Fig. 2 Block diagram of HPLC system detector, EC-8 (Toyo Soda, Tokyo, Japan) to identify the peaks (applied voltage was $0.6 \mathrm{~V}$ or $0.9 \mathrm{~V})$. Metabolites in kynurenine pathways were detected at $350 \mathrm{~nm}$ with a model UV-8 spectrophotometer (Toyo Soda, Tokyo, Japan). Native fluorescence detection (Ex. $370 \mathrm{~nm}, E \mathrm{~m} .470 \mathrm{~nm}$ or Ex. $330 \mathrm{~nm}, E m .380 \mathrm{~nm}$ ) was also used to identify Kyn or KA.

\section{Chromatographic condition}

Plasma or serum sample was mixed with $1 / 30$ volume of $20 \%$ hydrogen chloride to adjust the $\mathrm{pH}$ at around 2 , and was injected onto the pre-column, which was equilibrated with $0.1 \mathrm{M}$ phosphate buffer (pH 2.0) containing $1.0 \%$ trichloroacetic acid (TCA) by pump A. Then, the column was washed with the same buffer in order to elute out plasma proteins and other hydrophilic compounds. The Trp-metabolites were trapped on the top of the pre-column. Then, the pre-column was connected in a manner of flow through mode with the analytical column to allow the trapped Trpmetabolites to be eluted through the analytical column. The adsorbed Trpmetabolites were separated with stepwise elution of 0.1 M phosphate solution containing $1.5 \%, 4 \%, 20 \%$ and $30 \%$ acetonitrile, successively, at $35^{\circ} \mathrm{C}$ by pump $\mathrm{B}$.

\section{RESULTS AND DISCUSSION}

\section{Comparison of plasma with serum}

Chromatograms of Trp-metabolites in rabbit plasma and serum are shown in Figs. 3-b and 3-c. Rabbit heparinized plasma sample showed the peaks corresponding to 5HT, 5-HIAA, ILA, IAA, IPA, Kyn and KA in addition to Trp. The presence of the indole compounds were also identified by electrochemical detection (applied voltage $0.6 \mathrm{~V}$ or $0.9 \mathrm{~V}$ ), and Kyn and $\mathrm{KA}$ by native fluorescence detection (Kyn, Ex. $370 \mathrm{~nm}$, Em. $470 \mathrm{~nm} ; \mathrm{KA}, \mathrm{Ex} .330 \mathrm{~nm}, \mathrm{Em} .380 \mathrm{~nm})$. These data were not shown. In general, serum levels of 5-HT, 5-HIAA and 5-OH TrpOL were higher than plasma levels. One of the reasons might be the release of 5-HT from platelets during serum preparation from whole blood. Accordingly, it seems to be reasonable that the serum level of 5-HT was higher than that of the plasma level, and that 5-HIAA and 5-OH TrpOL which are 

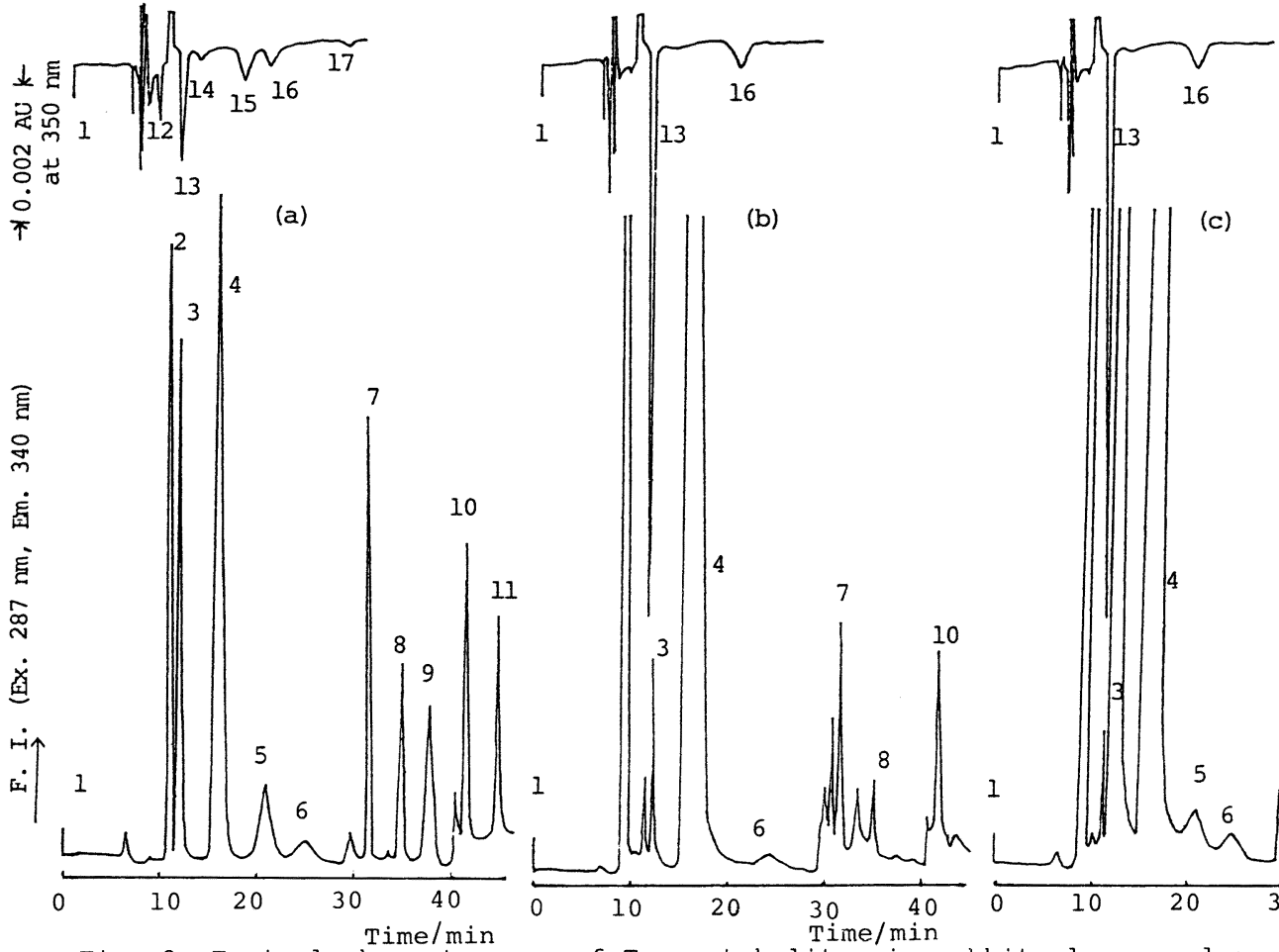

Fig. 3 Typical chromatograms of Trp-metabolites in rabbit plasma and serum

Samples: (a) standará metabolites (Trp 1 nmol; other indole metabolites 100 pmol; metabolites in kynurenine pathways 200 pmol)

(b) rabbit plasma $(100 \mu l)$

(c) rabbit serum $(100 \mu l)$

Elution: 1) $0.1 \mathrm{M}$ phosphate buffer ( $\mathrm{pH} 2.0$ ) containing $1.08 \mathrm{TCA}, 6 \mathrm{~min} 0.9 \mathrm{ml} / \mathrm{min}$

2) $0.1 \mathrm{M}$ phosphate solution ( $\mathrm{pH} 3.3$ ) containing $1.58 \mathrm{CH}_{3} \mathrm{CN}, 1 \mathrm{~min} 1.3 \mathrm{ml} / \mathrm{min}$

3) $0.1 \mathrm{M}$ phosphate solution ( $\mathrm{pH} 3.3$ ) containing $4.08 \mathrm{CH}_{3} \mathrm{CN}, 23 \mathrm{~min} 1.3 \mathrm{ml} / \mathrm{min}$

4) $0.1 \mathrm{M}$ phosphate solution $(\mathrm{pH} 4.5)$ containing $208 \mathrm{CH}_{3} \mathrm{CN}, 10 \mathrm{~min} 1.3 \mathrm{ml} / \mathrm{min}$

5) $0.1 \mathrm{M}$ phosphate solution $(\mathrm{pH} 4.5)$ containing $30 \% \mathrm{CH}_{3} \mathrm{CN}, 10 \mathrm{~min} 1.3 \mathrm{ml} / \mathrm{min}$

Detection: metabolites in indole pathways; native fluorescence (Ex $287 \mathrm{~nm}, \mathrm{Em} 340 \mathrm{~nm}$ ) metabolites in kynurenine pathways; UV spectrophotometry at $350 \mathrm{~nm}$

Signals: 1 ; injection marker, 2; 5-OH Trp, 3; 5-HT, 4; Trp, 5; 5-OH TrPOL, 6; 5-HIAA

7; ILA, 8; IAA, 9; TrPOL (tryptophol), 10; IPA, 11; IBA (indole-3-butylic acid: internal standard), 12; 3-OH Kyn, 13; KYn, 14;3-OH AA, 15; XA, 16; KA, 17; AA

metabolites from 5-HT (see Fig. 1) were also higher.

Furthermore, it was difficult to obtain the same serum levels of 5-HT, 5-HIAA and 5-OH TrpOL from the same rabbit. The reason might be the different degree or quantity of platelets involved in serum preparation. Thus, it was concluded that serum samples were not suitable for the study of Trp-metabolites in biological processes. 


\section{Stability of whole blood}

Whole heparinized rabbit blood was stood at $37^{\circ} \mathrm{C}$, and an aliquot was periodically taken out, and after centrifugation at $1500 \times \mathrm{g}$ for $10 \mathrm{~min}$ at room temperature, the Trp-metabolites level in plasma was analyzed. As shown in Fig. 4a, with increasing standing time as whole blood, the plasma levels of almost all of the Trp-metabolites, including metabolites in kynurenine pathways, were varied rapidly. The reason might be complicated, but at least the standing time as whole blood should be as short as possible.

\section{Stability of PRP}

As plasma contains a small number of platelets oftenly, the effect of platelets on Trp-metabolites level in plasma was studied by using platelet rich plasma (PRP) as the model sample. As shown in Fig. 4-b, with increasing standing time as PRP at $37^{\circ} \mathrm{C}$, the plasma levels of 5-HIAA and 5-OH TrpOL increased proportionally. These phenomena might be the result of enzymatic conversion of 5-HT, which was released from platelets. In PRP, the 5-OH TrPOL level was higher than 5-HIAA in contrast to the case of whole blood, and a possible reason might be due to the difference of enzymatic activities involved. The reason for 5-HT level fluctuation (the similar phenomenon was also observed with whole blood) was not clear. At any rate, there was a trend that platelets released 5-HT, followed by conversion to 5-OH TrpOL and 5HIAA. Accordingly, the plasma sample should contain as little platelets as possible, especially when the plasma sample has to be stored long time. The plasma containing platelets should not be stored in a freezer, because the platelets will be destroyed in a frozen state. The best way to obtain platelet poor plasma is to re-centrifuge the plasma sample mentioned in "method" section at the same condition.

\section{Stability of plasma}

In rabbit heparinized plasma sample, which was separated from blood cells just after blood collecting, the Trp-metabolites levels except 5-HT was constant for at least $24 \mathrm{~h}$ when it was stood at $37^{\circ} \mathrm{C}$ in contrast to PRP or whole blood. The 5-HT level showed the trend to decrease with time (ca. 5\%/h) and after the disappearance of 5-HT, no significant 5-HIAA or 5-OH Trpol peak was detected. In preliminary experiment, the addition of pargiline (monoamineoxidase inhibitor; $200 \mu \mathrm{g} / \mathrm{ml} \mathrm{plasma}$ ) or ascorbic acid (anti-oxidant; $30 \mu \mathrm{g} / \mathrm{ml}$ plasma) gave little or no effect. Rat heparinized plasma containing pargiline showed weak or no 5-HT degradative activity. The investigation of protection procedure for $5-\mathrm{HT}$ in rabbit plasma is under progress in our laboratory.

\section{Differences in animal source}

In preliminary experiment, it was shown that, in contrast to rabbit blood, rat heparinized blood had no detectable activity of the formation of 5-HIAA or 5-OH TrpOL from 5-HT, and that rat heparinized plasma showed weak or no 5-HT degradative activity in the presence of pargiline. These data indicate that enzymatic activities should be carefully checked before the analysis of Trp-metabolites level in plasma in each species of animals. 


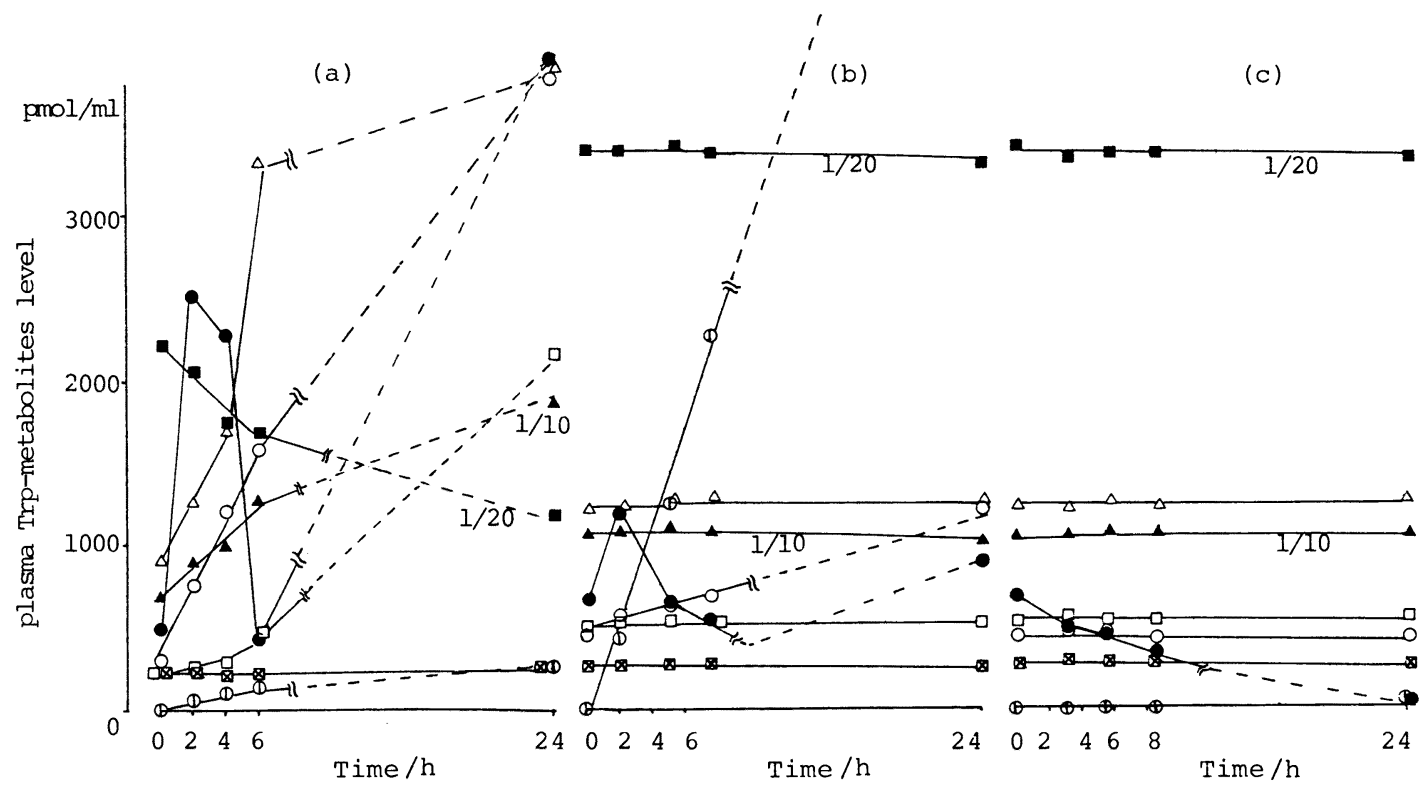

Fig. 4 Effects of standing time of (a) whole blood, (b) PRP and (c) plasma on Trp-metabolites

All samples were stood at $37^{\circ} \mathrm{C}$ during this study.

Rabbit blood samples for (b) and (c) were the same origin, but for (a) was different.

Symbols; - 5-HT, O 5-HIAA, (1) 5-OH TrPOL, -Trp, ILA, IAA, $\Delta$ Kyn, $\triangle$ KA

This research was supported in part by a Grant-in-Aid for Scientific Research from the Iatro Chemical Foundation.

\section{REFERENCES}

1) D. J. Boullin: "Serotonin in Mental Abnormalities", (1978), (Willey, New York).

2) G. Curzon, P. K. Bridges : J. Neurol. Neurosurg. Phychiatry., 33, 698 (1970).

3) H. Yoshida, I. Morita, T. Masujima, H. Imai : Chem. Pharm. Bull., 30, 2278 (1982).

4) H. Yoshida, I. Morita, T. Masujima, H. Imai : Chem. Pharm. Bull., 30, 3327 (1982).

5) I. Morita, T. Masujima, H. Yoshida, H. Imai : manuscript in preparation. (Abstract of 32 nd Annual Meeting of Jpn. Soc. Anal. Chem., p. 468 (1983)).

6) H. Yoshida, K. Takano, I. Morita, T. Masujima, H. Imai : Jpn. J. Clin. Chem., 12, 312, (1983).

Keyword phases

direct plasma injection method for HPLC analysis; variation of tryptophan metabolites level with sample preparation procedures; tryptophan metabolites in kynurenine and indole pathways. 